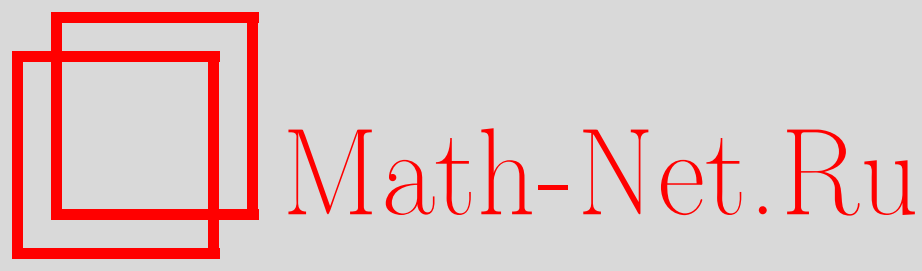

Ф. Дараби, Ф. Фелегари, Голографические модели темной энергии со взаимодействием и поправками к энтропии при различных обрезаниях в космологии БрансаДикке, ТМФ, 2017, том 191, номер 2, 334-353

DOI: https://doi.org/10.4213/tmf9199

Использование Общероссийского математического портала Math-Net.Ru подразумевает, что вы прочитали и согласны с пользовательским соглашением http://www . mathnet.ru/rus/agreement

Параметры загрузки:

IP: 3.80 .253 .173

26 апреля 2023 г., 13:01:45

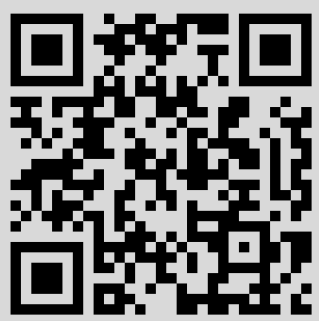




\title{
ФИЗИКА
}

Том 191, № 2

май, 2017

(C) 2017 r.

Ф. Дараби*, Ф. Фелегари*

\section{ГОЛОГРАФИЧЕСКИЕ МОДЕЛИ ТЕМНОЙ ЭНЕРГИИ СО ВЗАИМОДЕЙСТВИЕМ И ПОПРАВКАМИ К ЭНТРОПИИ ПРИ РАЗЛИЧНЫХ ОБРЕЗАНИЯХ В КОСМОЛОГИИ БРАНСА-ДИККЕ}

\begin{abstract}
В рамках космологии Бранса-Дикке изучаются модели голографической темной энергии со взаимодействием и со степенными и логарифмическими поправками к энтропии при различных обрезаниях. Для демонстрации того, в каких из моделей с поправками к энтропии могут иметь место ускорение и фантомная фаза в ранней Вселенной и в настоящем, условия на параметр Бранса-Дикке рассматриваются в сопоставлении с условиями ускорения Вселенной и наличия фантомной фазы. Кроме того, определяется, какие из рассматриваемых моделей являются классически устойчивыми и какие - неустойчивыми, при ранних временах и в настоящем.
\end{abstract}

Ключевые слова: параметр Бранса-Дикке, поправки к энтропии, голографическая темная энергия.

DOI: https://doi.org/10.4213/tmf9199

\section{1. ВВЕДЕНИЕ}

Недавние космологические и астрофизические данные, полученные по наблюдениям сверхновых типа Іа, реликтового излучения и крупномасштабной структуры Вселенной, дают серьезные основания полагать, что наблюдаемая Вселенная переживает фазу расширения [1], и это расширение может управляться таинственной составляющей энергии с отрицательным давлением - так называемой темной энергией. Космологическая постоянная является самым простым кандидатом на роль темной энергии, это называется моделью $\Lambda$ CDM. На сегодняшний день в космологии известно, что введение космологической постоянной сталкивается с двумя проблемами - тонкой настройки и космических совпадений [2], [3]. Проблема космических совпадений заключается в том, что Вселенная ведет себя так, что отношение плотностей темной материи и темной энергии должно быть постоянной

${ }^{*}$ Department of Physics, Azarbaijan Shahid Madani University, Tabriz, Iran.

E-mail: f.darabi@azaruniv.ac.ir, falegari@azaruniv.ac.ir 
порядка единицы [4]. Существуют различные модели темной энергии, в том числе модели, в которых темная энергия есть космологическая постоянная, квинтэссенция, $k$-эссенция, фантомная энергия, квинтом, газ Чаплыгина, тахион и модифицированная гравитация [5], [2]. Недавно в работе [6] в качестве новой модели, основанной на голографическом принципе, была предложена модель голографической темной энергии (ГТЭ):

$$
\rho_{\Lambda}=3 c^{2} M_{\mathrm{P}}^{2} L^{-2}
$$

где $c$ - постоянная, $L$ - радиус обрезания и $M_{\mathrm{P}}$ - редуцированная планковская масса. В модели ГТЭ важную роль играет энтропийное соотношение Бекенштейна-Хокинга

$$
S_{\mathrm{BH}}=\frac{A}{4 G},
$$

которое выполняется на горизонте событий [7]; здесь $A \sim L^{2}$ - площадь горизонта. Поскольку это соотношение тесно связано с законом пропорциональности площади для энтропии, любые поправки к этому закону влияют на плотность энергии в модели ГТЭ. Такие поправки могут возникать из квантовой теории поля [8], [9], термических и квантовых флуктуаций в Большой группе квазаров [10]-[14], а также из теории струн [15].

Одной из возможных поправок к соотношению для энтропии является степенная поправка [8]:

$$
S=\frac{A}{4 G}\left(1-K_{\alpha} A^{1-\alpha / 2}\right)
$$

здесь $\alpha$ - безразмерная константа и

$$
K_{\alpha}=\frac{\alpha(4 \pi)^{\alpha / 2-1}}{(4-\alpha) r_{\mathrm{c}}^{2-\alpha}},
$$

где $r_{\mathrm{c}}$ - масштаб кроссовера. Эта поправка приводит к модификации модели ГТЭ, которая в работе [16] названа моделью ГТЭ с полиномиальной поправкой к энтропии (модель ГТЭППЭ):

$$
\rho_{\Lambda}=3 c^{2} M_{\mathrm{P}}^{2} L^{-2}-\beta M_{\mathrm{P}}^{2} L^{-\alpha},
$$

где $\alpha$ и $\beta$ - две константы порядка единицы. В работе [8] было показано, что обобщенный второй закон термодинамики для Вселенной со степенной поправкой к энтропии выполняется при $\alpha>2$. Если $\alpha>2$, то второе слагаемое в (4) сравнимо по порядку величины с первым слагаемым, когда $L$ очень мало. Таком образом, поправка имеет физический смысл только в ранней Вселенной и пренебрежимо мала, когда Вселенная становится большой [16].

Еще одна возможная поправка к энтропии имеет следующий вид [17]:

$$
S=\frac{A}{4 G}+\tilde{\alpha} \ln \frac{A}{4 G}+\tilde{\beta},
$$

где $\tilde{\alpha}$ и $\tilde{\beta}$ - две безразмерные константы. Логарифмический член также возникает в модели энтропийной космологии [18], в рамках которой можно объединить инфляцию Вселенной на ранних временах и ее ускорение на поздних временах. Соотношение площадей для энтропии можно разложить в бесконечный ряд, однако вкладом дополнительных членов можно пренебречь из-за малости константы Планка $ћ$. 
Следовательно, в качестве наиболее важного лидирующего слагаемого остается логарифмический член, который и рассматривается в настоящей статье. Рассматривая модифицированное соотношение (5) для энтропии и учитывая изменение ГТЭ, мы модифицируем выражение для плотности энергии. Недавно в работе [19] было предложено записать плотность энергии $\rho_{\Lambda}$ для модели ГТЭ с логарифмической поправкой к энтропии (модель ГТЭЛПЭ) в следующем виде:

$$
\rho_{\Lambda}=\frac{3 c^{2} \phi^{2}}{4 \omega L^{2}}+\frac{\alpha}{L^{4}} \ln \frac{L^{2} \phi^{2}}{4 \omega}+\frac{\beta}{L^{4}},
$$

где $\alpha$ и $\beta$ - безразмерные константы. Второе и третье слагаемые в (6) сравнимы по величине с первым, только если $L$ очень мала, следовательно, поправка, вносимая этими слагаемыми, имеет физический смысл только в ранней Вселенной. Когда Вселенная становится большой, этими поправками можно пренебречь и ГТЭЛПЭ сводится к обычной ГТЭ. Поэтому в дальнейшем мы сначала исследуем сегодняшнее состояние Вселенной и затем изучаем эффект от различных обрезаний, рассматривая поправки к состоянию Вселенной на ранних временах [20]. Поскольку плотность ГТЭ отвечает динамической космологической постоянной, в качестве альтернативы общей теории относительности нам потребуется динамический подход, который согласован с такой плотностью ГТЭ.

Скалярно-тензорная теория гравитации как альтернатива общей теории относительности была впервые представлена в работах [21], [22]. Впоследствии она сыграла значительную роль в разрешении проблем стандартной космологии в контексте инфляционного сценария. Основная мотивация для использования моделей скалярного поля при поисках решения современных проблем темной энергии в космологии вытекает из физики элементарных частиц, а также из теории струн. Для решения проблемы темной энергии в рамках теории скалярного поля был предложен ряд моделей динамической темной энергии с эволюционирующим во времени уравнением состояния: модель квинтэссенции [23], $k$-эссенции [24], фантомной энергии [25], тахионные [26] и квинтомные [27] модели. Также одним из наиболее привлекательных предметов исследования в этом направлении является переформулировка голографических и алгебраических моделей темной энергии со скалярным полем [28]-[31]. Одна из таких попыток с интересными результатами - это исследование модели ГТЭ в рамках теории Бранса-Дикке (БД), в рамках которой, например, получается [32], что из модели ГТЭ для Вселенной, не являющейся плоской, нельзя получить уравнение состояния типа уравнения с фантомной энергией.

Цель настоящей работы - обобщить анализ, проведенный в работах [32], и рассмотреть в рамках космологии БД модели ГТЭ, содержащие взаимодействие и степенные или логарифмические поправки к энтропии. Мы получаем параметр уравнений состояния при различных подходящих обрезаниях. Мы также рассматриваем соответствие между, с одной стороны, моделями ГТЭ со взаимодействием и степенными и логарифмическими поправками к энтропии в космологии БД и, с другой стороны, моделями квинтэссенции и тахионными моделями скалярного поля для Вселенной, не являющейся плоской. Кроме того, мы проводим анализ устойчивости моделей, вводя в качестве определяющего параметра квадрат скорости звука $v_{\mathrm{s}}^{2}=d p / d \rho[33]$. Если $v_{\mathrm{s}}^{2}<0$, мы имеем классическую неустойчивость по отношению к возмущениям. Напротив, условие $v_{\mathrm{s}}^{2}>0$ может привести к устойчивой по отношению к возмущениям Вселенной. 


\section{2. МОДЕЛИ ТЕМНОЙ ЭНЕРГИИ СО ВЗАИМОДЕЙСТВИЕМ И ПОПРАВКАМИ К ЭНТРОПИИ В КОСМОЛОГИИ БД}

Действие в теории БД может быть записано в следующем каноническом виде [34]:

$$
\int d^{4} x \sqrt{g}\left(-\frac{1}{8 \omega} \phi^{2} R+\frac{1}{2} g^{\mu \nu} \partial_{\mu} \phi \partial_{\nu} \phi+L_{\mathrm{M}}\right),
$$

где $g$ - определитель тензорной метрики $g^{\mu \nu}, \omega$ - параметр БД, $R$ - скалярная кривизна Риччи и $L_{\mathrm{M}}-$ лагранжиан полей материи. Вариация действия по метрике $g^{\mu \nu}$ и скалярному полю БД $\phi$ дает

$$
\begin{gathered}
\phi G_{\mu \nu}=-8 \pi T_{\mu \nu}^{\mathrm{M}}-\frac{\omega}{\phi}\left(\phi_{, \mu} \phi_{, \nu}-\frac{1}{2} g_{\mu \nu} \phi_{, \lambda} \phi_{, \lambda}-\phi_{; \mu ; \nu}+\square \phi g_{\mu \nu}\right), \\
\square \phi=\frac{8 \pi}{2 \omega+3}\left(T^{\mathrm{M}}\right)_{\lambda}^{\lambda} .
\end{gathered}
$$

Здесь $T_{\mu \nu}^{\mathrm{M}}$ - тензор энергии-импульса полей материи.

Вселенная Фридмана-Робертсона-Уокера задается метрикой

$$
d s^{2}=d t^{2}-a^{2}(t)\left(\frac{d r^{2}}{1-k r^{2}}+r^{2} d \Omega^{2}\right)
$$

где $a(t)$ - масштабный фактор и $k$ - параметр кривизны. Используя это соотношение, мы можем привести полевые уравнения (8) и (9) к более простому виду

$$
\begin{gathered}
\frac{3}{4 \omega} \phi^{2}\left(H^{2}+\frac{k}{a^{2}}\right)-\frac{1}{2} \dot{\phi}^{2}+\frac{3}{2 \omega} H \dot{\phi} \phi=\rho_{\mathrm{m}}+\rho_{\Lambda}, \\
-\frac{1}{4 \omega} \phi^{2}\left(2 \frac{\ddot{a}}{a}+H^{2}+\frac{k}{a^{2}}\right)-\frac{1}{\omega} H \dot{\phi} \phi-\frac{1}{2 \omega} \ddot{\phi} \phi-\frac{1}{2}\left(1+\frac{1}{\omega}\right) \dot{\phi}^{2}=p_{\Lambda}, \\
\ddot{\phi}+3 H \dot{\phi}-\frac{3}{2 \omega}\left(\frac{\ddot{a}}{a}+H^{2}+\frac{k}{a^{2}}\right) \phi=0,
\end{gathered}
$$

где $H=\dot{a} / a$ - параметр Хаббла, $\rho_{\mathrm{m}}$ - плотность материи при нулевом давлении и $p_{\Lambda}-$ давление темной энергии.

Мы полагаем, что присутствует взаимодействие между ГТЭ с поправкой к энтропии и темной материей, которое задается следующим образом:

$$
\begin{aligned}
\dot{\rho}_{\Lambda}+3 H\left(1+\omega_{\Lambda}\right) \rho_{\Lambda} & =-Q, \\
\dot{\rho}_{M}+3 H \rho_{M} & =Q,
\end{aligned}
$$

где $Q=3 b^{2} H\left(\rho_{\Lambda}+\rho_{\mathrm{m}}\right)$ - член взаимодействия и $b^{2}$ - константа связи. Кроме того, следуя работам [35], мы предполагаем, что поле БД ведет себя, как $\phi=a^{n}$, таким образом,

$$
\dot{\phi}=n H \phi, \quad \ddot{\phi}=\left(n^{2} H^{2}+n \dot{H}\right) \phi .
$$


Особый интерес представляет случай, когда $n$ мало, а параметр $\omega$ велик, так что произведение $n \omega \sim 1$ [35]. Относительные плотности энергии задаются как

$$
\begin{aligned}
\Omega_{\mathrm{M}} & =\frac{4 \omega \rho_{\mathrm{m}}}{3 \phi^{2} H^{2}}, \\
\Omega_{k} & =\frac{k}{a^{2} H^{2}}, \\
\Omega_{\Lambda} & =\frac{4 \omega \rho_{\Lambda}}{3 \phi^{2} H^{2}} .
\end{aligned}
$$

Взяв производную по времени от уравнения (11), получим

$$
H(z)=H_{0}\left[\frac{\Omega_{M_{0}}(1+z)^{2 n+3}}{1+\Omega_{k}+(2 n / 3)(3-n \omega)-\Omega_{\Lambda}}\right]^{1 / 2},
$$

где мы преобразовали производную по времени в производную по $z$, а нижний индекс 0 означает, что данная величина рассматривается в настоящий момент времени. Также мы получаем параметр, характеризующий замедление:

$$
q=-\frac{\ddot{a} a}{\dot{a}^{2}}=-1-\frac{\dot{H}}{H^{2}} .
$$

Дифференцирование выражения (18) по времени дает

$$
\frac{d \Omega_{k}}{d z}=2 \Omega_{k}\left((1+z)^{-1}-\frac{d H / d z}{H}\right),
$$

где мы использовали соотношения

$$
\frac{d \Omega_{k}}{d t}=-H(1+z) \frac{d \Omega_{k}}{d z}, \quad \frac{d H}{d t}=-H(1+z) \frac{d H}{d z} .
$$

С другой стороны, дифференцируя выражение (19), с учетом уравнения (14) и формулы

$$
\frac{d \Omega_{\Lambda}}{d t}=-H(1+z) \frac{d \Omega_{\Lambda}}{d z}
$$

мы получаем

$$
\frac{d \Omega_{\Lambda}}{d z}=(1+z)^{-1}\left[3 b^{2}\left(\Omega_{\Lambda}+\Omega_{\mathrm{m}}\right)+3\left(1+\omega_{\Lambda}\right) \Omega_{\Lambda}+2 n \Omega_{\Lambda}-2 \Omega_{\Lambda}(1+z) \frac{d H / d z}{H}\right] .
$$

Дифференцируя выражение (20), с учетом уравнения (22) и формулы для $d H / d t$ из (23) мы получаем

$$
\begin{aligned}
\frac{d H / d z}{H}= & -\frac{\left(2 n+3+3 b^{2}\right)\left(1+\Omega_{k}-\left(2 n^{2} / 3\right) \omega+2 n\right)}{2(1+z)\left(-1+\left(2 n^{2} / 3\right) \omega-2 n\right)}- \\
& -\frac{-2 \Omega_{k}+3 \Omega_{\Lambda} \omega_{\Lambda}}{2(1+z)\left(-1+\left(2 n^{2} / 3\right) \omega-2 n\right)} .
\end{aligned}
$$


Следовательно, используя это равенство, мы можем записать для параметра замедления выражение

$$
q=-1-\frac{\left(2 n+3+3 b^{2}\right)\left(1+\Omega_{k}-\left(2 n^{2} / 3\right) \omega+2 n\right)-2 \Omega_{k}+3 \Omega_{\Lambda} \omega_{\Lambda}}{2\left(-1+\left(2 n^{2} / 3\right) \omega-2 n\right)} .
$$

Кроме того, применяя формулы (4), (6) и (19), получаем соотношения

$$
\begin{gathered}
\beta=\frac{3 c^{2}}{L^{-\alpha+2}}\left(1-\frac{L^{2} H^{2} \Omega_{\Lambda}}{c^{2}}\right), \\
\alpha \ln \frac{\phi^{2} L^{2}}{4 \omega}+\beta=L^{4}\left(H^{2} \Omega_{\Lambda}-\frac{c^{2}}{L^{2}}\right) \frac{3 \phi^{2}}{4 \omega},
\end{gathered}
$$

которые в дальнейшем будут использованы при выводе выражения для $\omega_{\Lambda}$.

Для моделей ГТЭ со степенной или логарифмической поправкой к энтропии при любых обрезаниях в космологии БД, беря производную выражения (4) с учетом (14) и производную выражения (6) с учетом (26), (27), мы получаем соответственно уравнения

$$
\begin{aligned}
\omega_{\Lambda}= & -1-\frac{b^{2}}{\Omega_{\Lambda}}\left(1+\Omega_{k}-\frac{2}{3} n^{2} \omega+2 n\right)- \\
& -\frac{2 c^{2}}{L^{2} H \Omega_{\Lambda}}\left[(1+z) \frac{d L / d z}{L}\left(1-\frac{\alpha}{2}\left(1-\frac{L^{2} H^{2} \Omega_{\Lambda}}{c^{2}}\right)\right)+\frac{L^{2} H^{2} \Omega_{\Lambda} n}{c^{2}}\right], \\
\omega_{\Lambda}= & -1-\frac{b^{2}}{\Omega_{\Lambda}}\left(1+\Omega_{k}-\frac{2}{3} n^{2} \omega+2 n\right)- \\
& -\left(n-\frac{d L / d z}{L}(1+z)\right)\left[\frac{2 c^{2}}{3 L^{2} H^{2} \Omega_{\Lambda}}+\frac{8 \alpha \omega(1+z)^{2 n}}{9 L^{4} H^{2} \Omega_{\Lambda}}\right]-\frac{4}{3}(1+z) \frac{d L / d z}{L} .
\end{aligned}
$$

При изучении эволюции Вселенной одной из наиболее важных величин является квадрат скорости звука $v_{\mathrm{s}}^{2}=d p / d \rho$. Для потока темной энергии он задается как

$$
v_{\mathrm{s}}^{2}=\frac{d p_{\Lambda}}{d \rho_{\Lambda}}=\frac{\dot{p_{\Lambda}}}{\dot{\rho_{\Lambda}}}
$$

Также мы имеем

$$
v_{\mathrm{s}}^{2}=\omega_{\Lambda}-\frac{\dot{\omega}_{\Lambda}}{3 H\left(1+\omega_{\Lambda}^{\mathrm{eff}}\right)}
$$

где $\omega_{\Lambda}^{\mathrm{eff}}=\omega_{\Lambda}+Q / 3 H \rho_{\Lambda}$ отвечает эффективному уравнению для параметра состояния темной энергии. Если $v_{\mathrm{s}}^{2}>0$, то мы имеем классическую устойчивость, а если $v_{\mathrm{s}}^{2}<0$, то мы имеем классическую неустойчивость Вселенной [36]. 


\section{3. МОДЕЛЬ ТЕМНОЙ ЭНЕРГИИ СО ВЗАИМОДЕЙСТВИЕМ И ПОПРАВКАМИ К ЭНТРОПИИ В СЛУЧАЕ ХАББЛОВСКОГО ОБРЕЗАНИЯ}

Хаббловское обрезание имеет вид $L=H^{-1}$.

3.1. Модель ГТЭППЭ. Используя соотношения (20), (24) и (28), получаем

$$
\begin{aligned}
& \omega_{\Lambda}=\left\{-1-\frac{b^{2}}{\Omega_{\Lambda}}\left(1+\Omega_{k}-\frac{2}{3} n^{2} \omega+2 n\right)-\frac{2 c^{2} H_{0}}{\Omega_{\Lambda}}\left[\frac{\Omega_{M_{0}}(1+z)^{2 n+3}}{1+\Omega_{k}+(2 n / 3)(3-n \omega)-\Omega_{\Lambda}}\right]^{1 / 2} \times\right. \\
& \left.\times\left[\left(\frac{\left(2 n+3+3 b^{2}\right)\left(1+\Omega_{k}-\left(2 n^{2} / 3\right) \omega+2 n\right)-2 \Omega_{k}}{2\left(-1-2 n+\left(2 n^{2} / 3\right) \omega\right)}\right)\left(1-\frac{\alpha}{2}\left(1-\frac{\Omega_{\Lambda}}{c^{2}}\right)\right)+\frac{n \Omega_{\Lambda}}{c^{2}}\right]\right\} \times \\
& \times\left\{\left(-1-2 n+\frac{2}{3} n^{2} \omega\right) \times\right. \\
& \left.\times\left[-1-2 n+\frac{2}{3} n^{2} \omega+3 c^{2} H_{0}\left(1-\frac{\alpha}{2}\left(1-\frac{\Omega_{\Lambda}}{c^{2}}\right)\right)\left[\frac{\Omega_{M_{0}}(1+z)^{2 n+3}}{1+\Omega_{k}+(2 n / 3)(3-n \omega)-\Omega_{\Lambda}}\right]^{1 / 2}\right]^{-1}\right\} .
\end{aligned}
$$

Мы полагаем, что в настоящий момент $z \simeq 0, \alpha \simeq 0, c^{2} \simeq 1.1$ [37], $n \simeq 0.005$ [38], $H_{0} \simeq 67.8, \Omega_{\Lambda} \simeq 0.7, \Omega_{M_{0}} \simeq 0.27, \Omega_{k} \simeq 0$ и $b^{2}=0.02$ [37]. Условие ускорения $q<0$ приводит к диапазону $-10^{4} \lesssim \omega \lesssim 10^{4}$, который практически совпадает с условием $\omega_{\Lambda}<-1$, при котором может существовать фантомная фаза.

Для ранней Вселенной, используя (21), (28) и условие $H \simeq$ const [39], мы получаем, что $q \simeq-1$ и $\omega_{\Lambda}=-1-2 n H$. При малых временах для выполнения неравенства $\omega_{\Lambda}<-1$ необходимо $n>0$, таким образом, значение $n \simeq 0.005$ [38] согласуется с наличием фантомной фазы. Следовательно, модель ГТЭППЭ с хаббловским обрезанием и при $-10^{4} \lesssim \omega \lesssim 10^{4}$ поддерживает сценарии инфляционной и ускоряющейся Вселенной с фантомной фазой для ранней и современной Вселенной соответственно.

Используя выражения для $\omega_{\Lambda}$ и ее производной по времени при малых временах и в настоящий момент, а также формулу $(31)$ с $b^{2}=0,0.02,0.04,0.06$, мы получаем для малых времен и в настоящий момент $v_{\mathrm{s}}^{2}<0$ и $v_{\mathrm{s}}^{2}>0$ соответственно. Это показывает, что модель ГТЭППЭ класически неустойчива при малых временах и классически устойчива в настоящем.

3.2. Модель ГТЭЛПЭ. Используя (20), (24) и (29), мы получаем

$$
\begin{aligned}
& \omega_{\Lambda}=\{--n\left[\frac{2 c^{2}}{3 \Omega_{\Lambda}}+\frac{8 \alpha \omega H_{0}^{2} \Omega_{M_{0}}(1+z)^{4 n+3}}{9 \Omega_{\Lambda}\left(1+\Omega_{k}+(2 n / 3)(3-n \omega)-\Omega_{\Lambda}\right)}\right]+ \\
&+ {\left[\frac{-2 \Omega_{k}+\left(2 n+3+3 b^{2}\right)\left(1+\Omega_{k}-\left(2 n^{2} / 3\right) \omega+2 n\right)}{2\left(-1+\left(2 n^{2} / 3\right) \omega-2 n\right)}\right] \times } \\
& \quad \times\left[\frac{2 c^{2}}{3 \Omega_{\Lambda}}+\frac{8 \alpha \omega H_{0}^{2} \Omega_{M_{0}}(1+z)^{4 n+3}}{9 \Omega_{\Lambda}\left(1+\Omega_{k}+(2 n / 3)(3-n \omega)-\Omega_{\Lambda}\right)}-\frac{4}{3}\right]- \\
&\left.-\frac{b^{2}}{\Omega_{\Lambda}}\left(1+\Omega_{k}-\left(2 n^{2} / 3\right) \omega+2 n\right)\right\} \times
\end{aligned}
$$

$\times\left[1-\left(\frac{3 \Omega_{\Lambda}}{2\left(-1+\left(2 n^{2} / 3\right) \omega-2 n\right)}\right)\left[\frac{2 c^{2}}{3 \Omega_{\Lambda}}+\frac{8 \alpha \omega H_{0}^{2} \Omega_{M_{0}}(1+z)^{4 n+3}}{9 \Omega_{\Lambda}\left(1+\Omega_{k}+(2 n / 3)(3-n \omega)-\Omega_{\Lambda}\right)}-\frac{4}{3}\right]\right]^{-1}$. 
Взяв тот же набор численных данных, что и выше, мы получаем условие $\omega \lesssim 8383$, для того чтобы получить в настоящем $q<0$. Однако для выполнения неравенства $\omega_{\Lambda}<-1$ требуется, чтобы $\omega \lesssim-2860$. Другими словами, неравенство $\omega \lesssim-2860$ поддерживает условия наличия фантомной фазы и ускоряющейся Вселенной. При $-2860 \lesssim \omega \lesssim 8380$ мы имеем ускоряющуюся Вселенную без фантомной фазы.

Для ранней Вселенной, используя (21), (29) и условие $H \simeq$ const, мы получаем, что $q \simeq-1$ и

$$
\omega_{\Lambda}=-1-n\left[\frac{2 c^{2}}{3 \Omega_{\Lambda}}+\frac{8 \alpha \omega H^{2}(1+z)^{2 n}}{9 \Omega_{\Lambda}}\right] .
$$

При значениях параметров $z \simeq 10^{4}, \alpha \simeq 1, c^{2} \simeq 1.1, \Omega_{\Lambda} \simeq 1$ для выполнения неравенств $\omega_{\Lambda}<-1$ или $\omega_{\Lambda}>-1$ требуется $\omega \gtrsim-H^{-2}$ или $\omega \lesssim-H^{-2}$ соответственно.

Таким образом, модель ГТЭЛПЭ с хаббловским обрезанием и $\omega \lesssim-2860$ поддерживает сценарии инфляции и ускоряющейся Вселенной с присутствием фантомной фазы в настоящей Вселенной; однако ранняя Вселенная может быть инфляционной и при этом иметь или не иметь фантомную фазу, если $\omega \gtrsim-H^{-2}$ или $\omega \lesssim-H^{-2}$ соответственно. Для модели ГТЭППЭ с использованием той же процедуры мы получаем $v_{\mathrm{s}}^{2}<0$ как для ранних, так и для настоящих времен, что объясняет классическую неустойчивость Вселенной в обеих эрах.

\section{4. ГОЛОГРАФИЧЕСКАЯ МОДЕЛЬ ТЕМНОЙ ЭНЕРГИИ СО ВЗАИМОДЕЙСТВИЕМ И ПОПРАВКАМИ К ЭНТРОПИИ В СЛУЧАЕ ОБРЕЗАНИЯ НА ВИДИМОМ ГОРИЗОНТЕ}

Обрезание на видимом горизонте задается масштабом

$$
L=\tilde{r}_{\mathrm{A}}=\frac{1}{\sqrt{H^{2}+k / a^{2}}} .
$$

4.1. Модель ГТЭППЭ. В случае голографической модели со степенной поправкой к энтропии, используя соотношения (20), (24), (28) и (33), мы можем получить параметр уравнения состояния для настоящего момента времени:

$$
\begin{aligned}
\omega_{\Lambda}=\{-1 & -\frac{b^{2}}{\Omega_{\Lambda}}\left(1+\Omega_{k}-\frac{2}{3} n^{2} \omega+2 n\right)- \\
- & \frac{2 c^{2} H_{0}\left(1+\Omega_{k}\right)}{\Omega_{\Lambda}}\left[\frac{\Omega_{M_{0}}(1+z)^{2 n+3}}{1+\Omega_{k}+(2 n / 3)(3-n \omega)-\Omega_{\Lambda}}\right]^{1 / 2} \times \\
& \times\left[\left(\left(2 n+3+3 b^{2}\right)\left(1+\Omega_{k}-\frac{2}{3} n^{2} \omega+2 n\right)-\right.\right. \\
& \left.-2 \Omega_{k}\left(-2 n+\frac{2}{3} n^{2} \omega\right)\right)\left(2\left(1+\Omega_{k}\right)\left(-1-2 n+\frac{2}{3} n^{2} \omega\right)\right)^{-1} \times \\
\times & \left.\left.\times\left(1-\frac{\alpha}{2}\left(1-\frac{\Omega_{\Lambda}}{c^{2}\left(1+\Omega_{k}\right)}\right)\right)+\frac{n \Omega_{\Lambda}}{c^{2}\left(1+\Omega_{k}\right)}\right]\right\} \times \\
& \left.+1-2 n+\frac{2}{3} n^{2} \omega\right)\left[-1-2 n+\frac{2}{3} n^{2} \omega+\right. \\
+ & \left.\left.3 c^{2} H_{0}\left(1-\frac{\alpha}{2}\left(1-\frac{\Omega_{\Lambda}}{c^{2}\left(1+\Omega_{k}\right)}\right)\right)\left[\frac{\Omega_{M_{0}}(1+z)^{2 n+3}}{1+\Omega_{k}+(2 n / 3)(3-n \omega)-\Omega_{\Lambda}}\right]^{1 / 2}\right]^{-1}\right\} .
\end{aligned}
$$


Мы полагаем, что в настоящий момент $z \simeq 0, \alpha \simeq 0, c^{2}=1.1$ [37], $n=0.005$ [38], $H_{0}=67.8, \Omega_{\Lambda}=0.7, \Omega_{M_{0}}=0.27, \Omega_{k} \simeq 0$ и $b^{2}=0.02$ [37]. При этих данных условие ускорения $q<0$ и присутствия фантомной фазы $\omega_{\Lambda}<-1$ приводят соответственно к неравенствам $\omega<10^{4}$ и $-10^{4}<\omega<10^{4}$. Таким образом, при $-10^{4}<\omega<10^{4}$ мы имеем как ускорение, так и фантомную фазу.

При ранних временах, используя (28) и условие $H=$ const, получаем, что $q \simeq-1$ и

$$
\omega_{\Lambda}=-1-\frac{2 c^{2} H\left(1+\Omega_{k}\right)}{\Omega_{\Lambda}}\left[-\frac{\Omega_{k}}{1+\Omega_{k}}\left(1-\frac{\alpha}{2}\left(1-\frac{\Omega_{\Lambda}}{c^{2}\left(1+\Omega_{k}\right)}\right)\right)+\frac{n \Omega_{\Lambda}}{c^{2}\left(1+\Omega_{k}\right)}\right] .
$$

Для выполнения условия $\omega_{\Lambda}<-1$ требуется $n>0$, поэтому величина $n \simeq 0.005$ [38] согласуется с наличием фантомной фазы. Следовательно, модель ГТЭППЭ с обрезанием на видимом горизонте и при $-10^{4} \lesssim \omega \lesssim 10^{4}$ поддерживает сценарии инфляционной и ускоряющейся Вселенной с фантомной фазой при ранних временах и в настоящем соответственно.

Используя выражения для $\omega_{\Lambda}$ и ее производной по времени, отвечающие ранним временам и настоящему моменту, а также формулу (31) с $b^{2}=0,0.02,0.04,0.06$, мы получаем для ранних времен и настоящего момента $v_{\mathrm{s}}^{2}<0$ и $v_{\mathrm{s}}^{2}>0$ соответственно. Это показывает, что модель ГТЭППЭ обладает классической неустойчивостью при ранних временах и классической устойчивостью в настоящем.

4.2. Модель ГТЭЛПЭ. Для модели ГТЭЛПЭ, используя (20), (24) и (29), для настоящего момента времени мы можем записать

$$
\begin{aligned}
& \omega_{\Lambda}=\left\{-1-\left(n+\frac{\Omega_{k}}{\left(1+\Omega_{k}\right)}\right)\left[\frac{2 c^{2}\left(1+\Omega_{k}\right)}{3 \Omega_{\Lambda}}+\frac{8 \alpha \omega H_{0}^{2} \Omega_{M_{0}}(1+z)^{4 n+3}\left(1+\Omega_{k}\right)^{2}}{9 \Omega_{\Lambda}\left(1+\Omega_{k}+(2 n / 3)(3-n \omega)-\Omega_{\Lambda}\right)}\right]+\right. \\
& +\frac{4 \Omega_{k}}{3\left(1+\Omega_{k}\right)}+ \\
& +\left[\frac{-2 \Omega_{k}+\left(2 n+3+3 b^{2}\right)\left(1+\Omega_{k}-\left(2 n^{2} / 3\right) \omega+2 n\right)}{2\left(-1+\left(2 n^{2} / 3\right) \omega-2 n\right)\left(1+\Omega_{k}\right)}\right] \times \\
& \times\left[\frac{2 c^{2}\left(1+\Omega_{k}\right)}{3 \Omega_{\Lambda}}+\frac{8 \alpha \omega H_{0}^{2} \Omega_{M_{0}}(1+z)^{4 n+3}\left(1+\Omega_{k}\right)^{2}}{9 \Omega_{\Lambda}\left(1+\Omega_{k}+(2 n / 3)(3-n \omega)-\Omega_{\Lambda}\right)}-\frac{4}{3}\right]- \\
& \left.-\frac{b^{2}}{\Omega_{\Lambda}}\left(1+\Omega_{k}-\frac{2 n^{2}}{3} \omega+2 n\right)\right\} \times \\
& \times\left\{1-\left(\frac{3 \Omega_{\Lambda}}{2\left(-1+\left(2 n^{2} / 3\right) \omega-2 n\right)\left(1+\Omega_{k}\right)}\right) \times\right. \\
& \left.\times\left[\frac{2 c^{2}\left(1+\Omega_{k}\right)}{3 \Omega_{\Lambda}}+\frac{8 \alpha \omega H_{0}^{2} \Omega_{M_{0}}(1+z)^{4 n+3}\left(1+\Omega_{k}\right)^{2}}{9 \Omega_{\Lambda}\left(1+\Omega_{k}+(2 n / 3)(3-n \omega)-\Omega_{\Lambda}\right)}-\frac{4}{3}\right]\right\}^{-1} .
\end{aligned}
$$

Для настоящего времени мы полагаем $z \simeq 0, c^{2}=1.1$ [37], $n=0.005$ [38], $H_{0}=67.8$, $\Omega_{M_{0}}=0.27, \Omega_{k} \simeq 0$ и $b^{2}=0.02$ [37]. При этих данных для $q<0$ и $\omega_{\Lambda}<-1$ мы получаем $-29032 \lesssim \omega \lesssim 34254$ и $16908 \lesssim \omega \lesssim 38858$ соответственно. Таким образом, ускорение и фантомная фаза присутствуют для некоторого диапазона значений $\omega$.

При ранних временах, используя (29), (33) и условие $H=$ const, мы получаем, что $q \simeq-1$ и

$$
\omega_{\Lambda}=-1-\left(n+\frac{\Omega_{k}}{\left(1+\Omega_{k}\right)}\right)\left[\frac{2 c^{2}\left(1+\Omega_{k}\right)}{3 \Omega_{\Lambda}}+\frac{8 \alpha \omega H^{2}(1+z)^{2 n}\left(1+\Omega_{k}\right)^{2}}{9 \Omega_{\Lambda}}\right]+\frac{4 \Omega_{k}}{3\left(1+\Omega_{k}\right)},
$$


где $\alpha$ - безразмерная константа порядка единицы. Для $z \simeq 10^{4}, \alpha \simeq 1, c^{2} \simeq 1.1$ и $\Omega_{\Lambda} \simeq 1$ условия $\omega_{\Lambda}<-1$ или $\omega_{\Lambda}>-1$ требуют выполнения неравенств $\omega \gtrsim-H^{-2}$ или $\omega \lesssim-H^{-2}$ соответственно. Таким образом, модель ЛТЭЛПЭ с обрезанием на видимом горизонте при $16908 \lesssim \omega \lesssim 38858$ поддерживает модель инфляционной и ускоряющейся Вселенной с фантомной фазой для настоящего времени, однако для ранней Вселенной мы можем получить инфляционные модели с фантомной фазой или без фантомной фазы, если $\omega \gtrsim-H^{-2}$ или $\omega \lesssim-H^{-2}$ соответственно.

Используя ту же процедуру, для модели ГТЭЛПЭ при настоящих временах мы получаем, что квадрат скорости звука $v_{\mathrm{s}}^{2}$ отрицателен при $0.5<\Omega_{\Lambda}<1$ и положителен при $0<\Omega_{\Lambda}<0.5$. Другими словами, мы имеем классическую неустойчивость при $0.5<\Omega_{\Lambda}<1$ и классическую неустойчивость при $0<\Omega_{\Lambda}<0.5$.

При ранних временах мы получаем, что квадрат скорости звука всегда отрицателен и имеет место классическая неустойчивость.

\section{5. МОДЕЛЬ ГТЭ С ПОПРАВКАМИ К ЭНТРОПИИ И ОБРЕЗАНИЕМ НА ГОРИЗОНТЕ СОБЫТИЙ}

Обрезание на горизонте событий задается соотношениями

$$
L=a(t) r(t), \quad r(t)=\frac{\sqrt{|k| y} \sin n}{\sqrt{|k|}}= \begin{cases}\sin y, & k=1, \\ y, & k=0 \\ \operatorname{sh} y, & k=-1\end{cases}
$$

где

$$
y=\frac{R_{\mathrm{h}}}{a(t)}=a(t) \int_{a(t)}^{\infty} \frac{d a(t)}{a^{2}(t) H} .
$$

Здесь $L$ - радиус горизонта событий, измеренный на сфере горизонта, и $R_{\mathrm{h}}$ - пространственный радиус горизонта событий [40].

5.1. Модель ГТЭППЭ. Для модели РГТЭППЭ, используя (20), (24), (28), (35) и (36), для настоящего момента мы можем записать выражение

$$
\begin{aligned}
\omega_{\Lambda}= & -1-\frac{b^{2}}{\Omega_{\Lambda}}\left(1+\Omega_{k}-\frac{2 n^{2}}{3} \omega+2 n\right)- \\
& -\frac{2 H_{0}}{\gamma_{\mathrm{c}}}\left[\frac{\Omega_{M_{0}}(1+z)^{2 n+3}}{1+\Omega_{k}+(2 n / 3)(3-n \omega)-\Omega_{\Lambda}}\right]^{1 / 2}\left[-1+n \gamma_{\mathrm{c}}+\sqrt{\frac{\Omega_{\Lambda}}{c^{2} \gamma_{\mathrm{c}}}-\Omega_{k}}\right],
\end{aligned}
$$

где

$$
\gamma_{\mathrm{c}}=1-\frac{\beta}{3 c^{2}} L^{2-\alpha}
$$

Для модели РГТЭППЭ при ранних временах, используя (28), (35), (36) и условие $H \simeq$ const, мы получаем, что $q \simeq-1$ и

$$
\omega_{\Lambda}=-1-2 H c^{2}\left[\left(-1+\sqrt{\Omega_{\Lambda}-\Omega_{k}}\right)\left(1-\frac{\alpha}{2}\left(1-\frac{1}{c^{2}}\right)\right)+\frac{n}{c^{2}}\right] .
$$

Для настоящего момента мы полагаем $z \simeq 0, \alpha \simeq 0, c^{2}=1.1$ [37], $n=0.005$ [38], $H_{0}=67.8, \Omega_{\Lambda}=0.7, \Omega_{M_{0}}=0.27, \Omega_{k} \simeq 0$ и $b^{2}=0.02$ [37]. При этих значениях 
параметров условие $q<0$ приводит к ограничению $-5000<\omega<0$; при этом нельзя получить никакие значения параметра Бранса-Дикке, которые гарантировали бы выполнение условия $\omega_{\Lambda}<-1$ наличия фантомной фазы. Следовательно, мы имеем ускорение без фантомной фазы.

Используя выражения для $\omega_{\Lambda}$ и ее производной по времени при ранних временах и в настоящем, а также формулу (31) с $b^{2}=0,0.02,0.04,0.06$, мы получаем в настоящем классическую устойчивость в диапазоне $0<\Omega_{\Lambda}<0.2$ и классическую неустойчивость в диапазоне $0.2<\Omega_{\Lambda}<1$. При ранних временах мы находим, что квадрат скорости звука $v_{\mathrm{s}}^{2}$ положителен, что свидетельствует о классической устойчивости.

5.2. Модель ГТЭЛПЭ. Используя (20), (24), (29), (35) и (36), для настоящего момента мы можем записать выражение

$$
\begin{aligned}
\omega_{\Lambda}= & -1-n\left[\frac{2}{3 \gamma_{\alpha}}+\frac{8 \alpha \omega(1+z)^{2 n}}{9 L^{2} c^{2} \gamma_{\alpha}}\right]+ \\
& +\left(1-\sqrt{\left.\frac{\Omega_{\Lambda}}{c^{2} \gamma_{\alpha}}-\Omega_{k}\right)}\left[-\frac{2}{3 \gamma_{\alpha}}-\frac{8 \alpha \omega(1+z)^{2 n}}{9 L^{2} c^{2} \gamma_{\alpha}}+\frac{4}{3}\right]-\frac{b^{2}}{\Omega_{\Lambda}}\left(1+\Omega_{k}-\frac{2 n^{2}}{3} \omega+2 n\right),\right.
\end{aligned}
$$

где

$$
\gamma_{\alpha}=1+\frac{4 \omega(1+z)^{2 n}}{3 L^{2} c^{2}}\left[\alpha \ln \left(\frac{L^{2}}{4 \omega(1+z)^{2 n}}+\beta\right)\right] .
$$

Для модели ГТЭЛПЭ при ранних временах, используя (29), (35), (36) и условие $H=$ const, мы получаем, что $q \simeq-1$ и

$\omega_{\Lambda}=-1-n\left[\frac{2 c^{2}}{3}+\frac{8 \alpha \omega(1+z)^{2 n} H^{2}}{9}\right]+\left(1-\sqrt{\Omega_{\Lambda}-\Omega_{k}}\right)\left[-\frac{2 c^{2}}{3}-\frac{8 \alpha \omega(1+z)^{2 n} H^{2}}{9}+\frac{4}{3}\right]$.

Для настоящего момента мы полагаем $z \simeq 0, \alpha \simeq 0, c^{2}=1.1$ [37], $n=0.005$ [38], $H_{0}=67.8, \Omega_{\Lambda}=0.7, \Omega_{M_{0}}=0.27, \Omega_{k} \simeq 0$ и $b^{2}=0.02$ [37]. При этих значениях параметров условия ускорения $q<0$ и наличия фантомной фазы $\omega_{\Lambda}<-1$ приводят к неравенствам $-51628 \lesssim \omega \lesssim 59086$ и $\omega \lesssim-225706$ соответственно. Следовательно, мы имеем ускорение без фантомной фазы.

При ранних временах, используя значения параметров $z \simeq 10^{4}, \alpha \simeq 1, c^{2} \simeq 1.1$ и $\Omega_{\Lambda} \simeq 1$, из $\omega_{\Lambda}<-1$ или $\omega_{\Lambda}>-1$ получаем $\omega \gtrsim-H^{-2}$ и $\omega \lesssim-H^{-2}$ соответственно.

Применяя ту же процедуру для модели ГТЭЛПЭ, мы получаем $v_{\mathrm{s}}^{2}<0$ в настоящем и $v_{\mathrm{s}}^{2}>0$ на ранних временах, что объясняет классическую неустойчивость в настоящем и классическую неустойчивость при ранних временах.

\section{6. МОДЕЛЬ ГТЭ СО ВЗАИМОДЕЙСТВИЕМ И ПОПРАВКАМИ К ЭНТРОПИИ ПРИ ОБРЕЗАНИИ С ПОМОЩЬЮ СКАЛЯРА РИЧЧИ}

Рассмотрим инфракрасное обрезание вида $L=R^{-1 / 2}$, где $R$ - скалярная кривизна Риччи. Обрезание с помощью скаляра Риччи задается формулой

$$
R=6\left(\dot{H}+2 H^{2}+\frac{k}{a^{2}}\right) .
$$


Здесь $\dot{H}$ - производная параметра Хаббла по космическому времени $t$. Используя соотношения (11) и (16), мы получаем [41]

$$
H^{2}+\frac{k}{a^{2}}=\frac{4 \omega}{3 \phi^{2}}\left(\rho_{\Lambda}+\rho_{\mathrm{M}}\right)+2 n H^{2}\left(-1+\frac{n \omega}{3}\right) .
$$

Теперь с учетом этих формул мы можем записать

$$
R=6\left\{\dot{H}+H^{2}+\frac{4 \omega}{3 \phi^{2}}\left(\rho_{\Lambda}+\rho_{\mathrm{M}}\right)+2 n H^{2}\left(-1+\frac{n \omega}{3}\right)\right\} .
$$

Продифференцируем равенство (38) по космическому времени $t$ и учтем соотношения (14), (15) и (38), отсюда выводим равенство

$$
\dot{H}+H^{2}=\frac{4 \omega}{3 \phi^{2}(-1+2 n(n \omega / 3-1))}\left[\rho_{\Lambda}\left(\frac{3}{2} \omega_{\Lambda}+\frac{1}{2}+n\right)+\left(n+\frac{1}{2}\right) \rho_{\mathrm{M}}\right] .
$$

Подставляя его в (39), имеем

$$
\begin{aligned}
\omega_{\Lambda}= & \left(\frac{\phi^{2}\left(2 n^{2} \omega-6 n-3\right)}{\rho_{\Lambda}}\right)\left(\frac{R}{36 \omega}-\frac{n H^{2}(n \omega-3)}{9 \omega}\right)- \\
& -\frac{\left(1+\Omega_{k}-\left(2 n^{2} / 3\right) \omega+2 n\right)\left(4 n^{2} \omega-6 n-3\right)}{9 \Omega_{\Lambda}} .
\end{aligned}
$$

Кроме того, используя $(12),(16),(19),(21)$ и выражение $P_{\Lambda}=\rho_{\Lambda} \omega_{\Lambda}$, мы находим

$$
q=\frac{1}{2(n+1)}\left[3 \Omega_{\Lambda} \omega_{\Lambda}+(2 n+1)^{2}+2 n(n \omega-1)+\Omega_{k}\right]
$$

6.1. Модель ГТЭППЭ. Используя формулу (4) и равенство $L=R^{-1 / 2}$, мы находим

$$
\rho_{\Lambda}=\frac{3 c^{2} \phi^{2} R}{4 \omega} \gamma_{\mu}, \quad \gamma_{\mu}=1-\frac{\beta}{3 c^{2}} R^{\alpha / 2-1}
$$

При ранних временах из соотношений (28), (37) и условия $H=$ const получаем, что $q \simeq-1$ и

$$
\omega_{\Lambda}=-1+\frac{12 c^{2} H}{\Omega_{\Lambda}}\left[-1+\frac{2 n^{2}}{3} \omega-2 n+\Omega_{\Lambda}\right]\left\{1-\frac{\alpha}{2}\left(1-\frac{H^{2} \Omega_{\Lambda}}{c^{2} R}\right)\right\}-2 n H .
$$

Здесь $\alpha$ - безразмерная константа порядка единицы. Мы полагаем, что при ранних временах $c^{2}=1.1, \Omega_{k} \simeq 0$ [37], и считаем, что $\alpha>2$ [16]. Оказывается, что для выполнения условия $\omega_{\Lambda}<-1$ требуется $n>0$ при любых $\omega$.

Для $0<R<1$ при ранних временах, взяв производную по времени от $\omega_{\Lambda}$ и использовав выражения (31) и (44), находим, что квадрат скорости звука при ранних временах всегда отрицателен, и это означает, что мы имеем классическую неустойчивость. 
6.2. Модель ГТЭЛПЭ. Используя выражение (6) и равенство $L^{2}=R^{-1}$, можно получить

$$
\rho_{\Lambda}=\frac{3 c^{2} \phi^{2} R}{4 \omega} \gamma_{\phi}, \quad \gamma_{\phi}=1+\frac{4 \omega R}{3 c^{2} \phi^{2}}\left(\alpha \ln \frac{\phi^{2}}{4 \omega R}+\beta\right) .
$$

При ранних временах из формул (37) и (29) и условия $H \simeq$ const мы получаем, что $q \simeq-1$ и

$$
\omega_{\Lambda}=-1-n\left[\frac{8 c^{2}}{\Omega_{\Lambda}}+\frac{128 \alpha \omega(1+z)^{2 n} H^{2}}{\Omega_{\Lambda}}\right],
$$

где $\alpha$ - безразмерная константа порядка единицы. Также мы полагаем $c^{2}=1.1$, $\Omega_{k} \simeq 0$ [37]. Потребовав выполнения условия $\omega_{\Lambda}<-1$, мы получаем диапазон $-10^{-98}<\omega<-10^{-42}$ и неравенство $n>-0.1$. Для $0<R<1$ при ранних временах, взяв производную по времени от выражения (46), с использованием соотношений (31) и (46) находим, что при ранних временах квадрат скорости звука всегда отрицателен. Это означает, что мы имеем классическую неустойчивость.

Для обеих моделей ГТЭППЭ и ГТЭЛПЭ при настоящих временах мы рассматриваем случай $\alpha=\beta=0, \gamma_{\mu}=1, \gamma_{\phi}=1$. Используя формулы $(20),(41),(43)$ и (45), получаем

$$
\begin{aligned}
\omega_{\Lambda}= & \left(\frac{4\left(2 n^{2} \omega-6 n-3\right)}{3 c^{2}}\right)\left[\frac{1}{36}-\frac{n H_{0}^{2}(n \omega-3) \Omega_{M_{0}}(1+z)^{2 n+3}}{9 R\left(1+\Omega_{k}+(2 n / 3)(3-n \omega)-\Omega_{\Lambda}\right)}\right]- \\
& -\frac{\left(1+\Omega_{k}-\left(2 n^{2} / 3\right) \omega+2 n\right)\left(4 n^{2} \omega-6 n-3\right)}{9 \Omega_{\Lambda}} .
\end{aligned}
$$

Теперь для обеих моделей ГТЭППЭ и ГТЭЛПЭ положим $c^{2}=1.1$ [37], $n=0.005$ [38], $H_{0}=67.8, \Omega_{M_{0}}=0.27, \Omega_{k} \simeq 0$ [37] и $0<R<1$. При этих значениях для выполнения условий $q<0$ и $\omega_{\Lambda}<-1$ имеем $2 \cdot 10^{4}<\omega<4.5 \cdot 10^{4}$ и $-10^{5}<\omega<10^{4}$ соответственно. Следовательно, мы наблюдаем ускорение без фантомной фазы.

Дифференцируя выражение (47) и используя для настоящего момента формулы (31) и $(47)$ при $b^{2}=0,0.02,0.04,0.06$ и $0<R<1$, находим, что квадрат скорости звука всегда положителен. Это означает, что мы имеем классическую устойчивость.

\section{7. МОДЕЛЬ ГТЭ СО ВЗАИМОДЕЙСТВИЕМ И С ПОПРАВКАМИ К ЭНТРОПИИ В СЛУЧАЕ ОБРЕЗАНИЯ ГРАНДЫ-ОЛИВЕРОСА}

Чтобы избежать проблемы причинности, Гранда и Оливерос предложили [29] новое обрезание для модели ГТЭ:

$$
L=\left(\tilde{\alpha} H^{2}+\tilde{\beta} \dot{H}\right)^{-1 / 2},
$$

где $\tilde{\alpha}$ и $\tilde{\beta}$ - константы. Подставляя (16) в (12) и используя выражение $P_{\Lambda}=\rho_{\Lambda} \omega_{\Lambda}$, получаем

$$
\rho_{\Lambda}=-\frac{\phi^{2} H^{2}}{4 \omega \omega_{\Lambda}}\left(3+\Omega_{k}+4 n+2 n^{2}(2+\omega)+\frac{\dot{H}}{H^{2}}(2 n+2)\right) .
$$


Кроме того, подставляя (24) в (48), мы находим

$$
\begin{aligned}
\omega_{\Lambda}= & {\left[3+\Omega_{k}+4 n+2 n^{2}(2+\omega)+\right.} \\
& \left.\quad+(n+1)\left(\frac{\left(2 n+3+3 b^{2}\right)\left(1+\Omega_{k}-\left(2 n^{2} / 3\right) \omega+2 n\right)-2 \Omega_{k}}{-1-2 n+\left(2 n^{2} / 3\right) \omega}\right)\right] \times \\
& \times\left(-\frac{4 \omega \rho_{\Lambda}}{\phi^{2} H^{2}}-\frac{3(n+1) \Omega_{\Lambda}}{-1-2 n+\left(2 n^{2} / 3\right) \omega}\right)^{-1} .
\end{aligned}
$$

Для моделей ГТЭППЭ и ГТЭЛПЭ, используя выражения (4) и (6), выводим соответственно

$$
\rho_{\Lambda}=\frac{3 c^{2} \phi^{2}}{4 \omega L^{2}} \gamma_{c} \quad \text { и } \quad \rho_{\Lambda}=\frac{3 c^{2} \phi^{2}}{4 \omega L^{2}} \gamma_{\alpha}
$$

где $L$ - масштаб обрезания Гранды-Оливероса. Для моделей ГТЭППЭ и ГТЭЛПЭ в настоящий момент времени, взяв $\alpha=\beta=0$ и использовав соотношения (20), (49) и (50), получим

$$
\begin{aligned}
\omega_{\Lambda}= & \left\{3+\Omega_{k}+4 n+2 n^{2}(2+\omega)+\right. \\
& \left.+(n+1)\left[\frac{\left(2 n+3+3 b^{2}\right)\left(1+\Omega_{k}-\left(2 n^{2} / 3\right) \omega+2 n\right)-2 \Omega_{k}}{-1-2 n+\left(2 n^{2} / 3\right) \omega}\right]\right\} \times \\
& \times\left\{-\frac{3 c^{2}\left(1+\Omega_{k}+(2 n / 3)(3-n \omega)-\Omega_{\Lambda}\right)}{\left.L^{2} H_{0}^{2} \Omega_{M_{0}}(1+z)^{2 n+3}-\frac{3(n+1) \Omega_{\Lambda}}{-1+\left(2 n^{2} / 3\right) \omega-2 n}\right\}^{-1},},\right.
\end{aligned}
$$

где $q$ задано в (42). Для настоящего времени возьмем $c^{2}=1.1$ [37], $n=0.005$ [38], $H_{0}=67.8, \Omega_{M_{0}}=0.27, \Omega_{k} \simeq 0$ и $b^{2}=0.02$ [37], а также $z=0$ и $0<L<1$. Для обеих моделей ГТЭППЭ и ГТЭЛПЭ условия $q<0$ и $\omega_{\Lambda}<-1$ приводят к ограничениям $10^{4}<\omega<1.2 \cdot 10^{4}$ и $1.11 \cdot 10^{4}<\omega<1.2 \cdot 10^{4}$ соответственно. Следовательно, ускорение и фантомную фазу мы можем получить при $1.11 \cdot 10^{4}<\omega<1.2 \cdot 10^{4}$.

При ранних временах, используя выражения (48), (49) и условие $H=$ const, получаем, что $q \simeq-1$ и

$$
\omega_{\Lambda}=-\frac{3+\Omega_{k}+4 n+2 n^{2}(2+\omega)}{3 \Omega_{\Lambda}} .
$$

Взяв $c^{2}=1.1$ и $\Omega_{k} \simeq 0$ [37], найдем, что требование $\omega_{\Lambda}<-1$ приводит к $n>0$.

Для настоящего времени, дифференцируя выражение для $\omega_{\Lambda}$ и используя формулу $(31)$ с $b^{2}=0,0.02,0.04,0.06$ и $0<L<1$, мы получаем классическую неустойчивость: для обеих моделей ГТЭППЭ и ГТЭЛПЭ квадрат скорости звука отрицателен. На ранних временах с помощью той же процедуры мы получаем аналогичный результат: для обеих моделей ГТЭППЭ и ГТЭЛПЭ мы имеем классическую неустойчивость. 


\section{8. РЕЗУЛЬТАТЫ}

Представим итоги наших рассмотрений в виде таблиц 1-6.

ТАБлицА 1. Ограничения для $\omega$, вытекающие из условия на параметр БД и условия $\omega_{\Lambda}<-1$ существования фантомной фазы, для настоящего времени.

\begin{tabular}{|l|c|c|}
\hline Обрезание & Модель ГТЭППЭ & Модель ГТЭЛПЭ \\
\hline хаббловское & $-10^{4}<\omega<10^{4}$ & $\omega \lesssim-2860$ \\
\hline на видимом горизонте & $-10^{4}<\omega<10^{4}$ & $16908 \lesssim \omega \lesssim 38858$ \\
\hline на горизонте событий & - & $\omega \lesssim-225706$ \\
\hline с помощью скаляра Риччи & $-10^{5}<\omega<-10^{4}$ & $-10^{5}<\omega<-10^{4}$ \\
\hline Гранды-Оливероса & $1.11 \cdot 10^{4}<\omega<1.2 \cdot 10^{4}$ & $1.11 \cdot 10^{4}<\omega<1.2 \cdot 10^{4}$ \\
\hline
\end{tabular}

ТАБлицА 2. Ограничения для $\omega$, вытекающие из условия на параметр БД и условия $q<0$ наличия ускорения, для настоящего времени.

\begin{tabular}{|l|c|c|}
\hline Обрезание & Модель ГТЭППЭ & Модель ГТЭЛПЭ \\
\hline хаббловское & $-10^{5}<\omega<10^{5}$ & $\omega \lesssim 8383$ \\
\hline на видимом горизонте & $-10^{5}<\omega<10^{5}$ & $-29032 \lesssim \omega \lesssim 34254$ \\
\hline на горизонте событий & $-5 \cdot 10^{3}<\omega<0$ & $-51628 \lesssim \omega \lesssim 59086$ \\
\hline с помощью скаляра Риччи & $2 \cdot 10^{4}<\omega<4.5 \cdot 10^{4}$ & $2 \cdot 10^{5}<\omega<4.5 \cdot 10^{5}$ \\
\hline Гранды-Оливероса & $10^{4}<\omega<1.2 \cdot 10^{4}$ & $10^{4}<\omega<1.2 \cdot 10^{4}$ \\
\hline
\end{tabular}

ТАБлицА 3. Ограничения для $\omega$, вытекающие из условия на параметр БД и условия $\omega_{\Lambda}<-1$ существования фантомной фазы, при ранних временах.

\begin{tabular}{|c|c|c|}
\hline Обрезание & Модель ГТЭППЭ & Модель ГТЭЛПЭ \\
\hline хаббловское & $\begin{array}{c}n>0, \\
-\infty<\omega<\infty\end{array}$ & $\omega>-H^{-2}$ \\
\hline на видимом горизонте & $\begin{array}{c}n>0, \\
-\infty<\omega<\infty\end{array}$ & $\omega>-H^{-2}$ \\
\hline на горизонте событий & $\begin{array}{c}n>0, \\
-\infty<\omega<\infty\end{array}$ & $\omega>-H^{-2}$ \\
\hline с помощью скаляра Риччи & $\begin{array}{c}n>0, \\
-\infty<\omega<\infty\end{array}$ & $\begin{array}{c}n>-0.1, \\
-10^{-98}<\omega<10^{-42}\end{array}$ \\
\hline Гранды-Оливероса & $n>0, \omega>0$ & $n>0, \omega>0$ \\
\hline
\end{tabular}


ТАБлицА 4. Ограничения для $\omega$, вытекающие из условия на параметр БД и условия $q<0$ наличия ускорения, при ранних временах.

\begin{tabular}{|c|c|c|}
\hline Обрезание & Модель ГТЭППЭ & Модель ГТЭЛПЭ \\
\hline хаббловское & $\begin{array}{c}n \neq 0,-3 / 2, \\
\omega=-\frac{3(3 H-2 n-4)}{n(2 n+3)}\end{array}$ & $\begin{array}{c}-\infty<n<\infty \\
\omega=\frac{6 n+8.7}{2 n^{2}+3 n+4 H^{2}(10001)^{2 n}}\end{array}$ \\
\hline $\begin{array}{l}\text { на видимом } \\
\text { горизонте }\end{array}$ & $\begin{array}{c}n \neq 0,-3 / 2, \\
\omega=-\frac{3(3 H-2 n-4)}{n(2 n+3)}\end{array}$ & $\begin{array}{c}-\infty<n<\infty \\
\omega=\frac{6 n+8.7}{2 n^{2}+3 n+4 H^{2}(10001)^{2 n}}\end{array}$ \\
\hline $\begin{array}{l}\text { на горизонте } \\
\text { событий }\end{array}$ & $\begin{array}{c}n \neq 0,-3 / 2, \\
\omega=-\frac{3(3 H-2 n-4)}{n(2 n+3)}\end{array}$ & $\begin{array}{c}-\infty<n<\infty \\
\omega=\frac{6 n+8.7}{2 n^{2}+3 n+4 H^{2}(10001)^{2 n}}\end{array}$ \\
\hline $\begin{array}{l}\text { с помощью } \\
\text { скаляра Риччи }\end{array}$ & $\begin{array}{c}n \neq 0, \\
\omega=\frac{33.75 H^{3}-10.5 H-1.25 n-1.25}{n\left(11.25 H^{3}-4.125 H+0.625\right)}\end{array}$ & $\omega=\frac{-\infty<n<\infty,}{6 n-33.6}$ \\
\hline Гранды-Оливероса & $n=0, \omega>0$ & $n=0, \omega>0$ \\
\hline
\end{tabular}

ТАБлицА 5. Классическая устойчивость или неустойчивость модели для настоящего времени.

\begin{tabular}{|c|c|c|}
\hline Обрезание & Модель ГТЭППЭ & Модель ГТЭЛПЭ \\
\hline хаббловское & устойчива & неустойчива \\
\hline на видимом горизонте & устойчива & $\begin{array}{c}\text { неустойчива } \\
\text { при } 0<\Omega_{\Lambda}<0.5, \\
\text { устойчива } \\
\text { при } 0.5<\Omega_{\Lambda}<1\end{array}$ \\
\hline на горизонте событий & $\begin{array}{c}\text { устойчива } \\
\text { при } 0<\Omega_{\Lambda}<0.2, \\
\text { неустойчива } \\
\text { при } 0.2<\Omega_{\Lambda}<1\end{array}$ & $\begin{array}{c}\text { неустойчива } \\
\text { сстойчива }\end{array}$ \\
\hline с помощью скаляра Риччи & устойчива & неустойчива \\
\hline Гранды-Оливероса & неустойчива & \\
\hline
\end{tabular}


ТАБлицА 6. Классическая устойчивость или неустойчивость модели при ранних временах.

\begin{tabular}{|l|c|c|}
\hline Обрезание & Модель ГТЭППЭ & Модель ГТЭЛПЭ \\
\hline хаббловское & неустойчива & неустойчива \\
\hline на видимом горизонте & неустойчива & неустойчива \\
\hline на горизонте событий & устойчива & устойчива \\
\hline с помощью скаляра Риччи & неустойчива & неустойчива \\
\hline Гранды-Оливероса & неустойчива & неустойчива \\
\hline
\end{tabular}

\section{9. ЗАКЛЮЧИТЕЛЬНЫЕ ЗАМЕЧАНИЯ}

В представленной работе мы изучили модель голографической темной энергии со взаимодействием и со степенными и логарифмическими поправками к энтропии при различных обрезаниях в рамках космологии Бранса-Дикке. Для сравнения моделей с двумя указанными поправками к энтропии при ранних временах и в настоящем мы получили параметр Бранса-Дикке и условия наличия ускорения и фантомной фазы. Кроме того, используя квадрат скорости света, мы сделали выводы о классической устойчивости или неустойчивости рассматриваемых моделей при различных обрезаниях. Этот анализ показывает, какие из моделей с поправками к энтропии могут проявлять себя как модели с ускорением и наличием или отсутствием фантомной фазы, а также какие модели являются устойчивыми и какие неустойчивыми, при ранних временах Вселенной и в настоящее время.

\section{Список литературы}

[1] A. G. Riess, A. V. Filippenko, P. Challis, A. Clocchiatti, A. Diercks, P. M. Garnavich, R. L. Gilliland, C. J. Hogan, S. Jha, R. P. Kirshner, B. Leibundgut, M. M. Phillips, D. Reiss, B. P. Schmidt, R. A. Schommer, R. Chris Smith, J. Spyromilio, C. Stubbs, N. B. Suntzeff, J. Tonry, "Observational evidence from Supernovae for an accelerating universe and a cosmological constant", Astron. J., 116:3 (1998), 1009-1038; S. Perlmutter, G. Aldering, G. Goldhaber et al. (The Supernova Cosmology Project), "Measurements of $\Omega$ and $\Lambda$ from 42 high-redshift Supernovae", Astrophys. J., 517:2 (1999), 565-586; P. de Bernardis, P. A. R. Ade, J. J. Bock et al., "A flat Universe from high-resolution maps of the cosmic microwave background radiation", Nature, 404:6781 (2000), 955-959; R. A. Knop, G. Aldering, R. Amanullah et al. (The Supernova Cosmology Project), "New Constraints on $\Omega_{M}$, $\Omega_{\Lambda}$, and $w$ from an independent set of 11 high-redshift Supernovae observed with the Hubble space Telescope", Astrophys. J., 598:1 (2003), 102-137; U. Seljak, A. Makarov, P. McDonald et al., "Cosmological parameter analysis including SDSS Ly $\alpha$ forest and galaxy bias: constraints on the primordial spectrum of fluctuations, neutrino mass, and dark energy", Phys. Rev. D, 71:10 (2005), 103515, 20 pp.

[2] E. J. Copeland, M. Sami, S. Tsujikawa, "Dynamics of dark energy", Internat. J. Modern Phys. D, 15:11 (2006), 1753-1935.

[3] S. Weinberg, "The cosmological constant problem", Rev. Modern Phys., 61:1 (1989), 1-23.

[4] W. Zimdahl, D. Pavon, "Interacting holographic dark energy", Class. Quantum Grav., 24:22 (2007), 5461-5478; Y. Bisabr, "Coincidence problem in $f(R)$ gravity models", Phys. Rev. D, 82:12 (2010), 124041, 6 pp. 
[5] T. Padmanabhan, "Cosmological constant - the weight of the vacuum", Phys. Rep., 380:5-6 (2003), 235-320; Y.-F. Cai, E. N. Saridakis, M. R. Setare, J.-Q. Xia, "Quintom cosmology: theoretical implications and observations", Phys. Rep., 493:1 (2010), 1-60.

[6] L. Susskind, "The world as a hologram", J. Math. Phys., 36:11 (1995), 6377-6396; S. Nojiri, S. D. Odintsov, "Unifying phantom inflation with late-time acceleration: scalar phantom-non-phantom transition model and generalized holographic dark energy", Gen. Relativ. Gravit., 38:8 (2006), 1285-1304; K. Bamba, S. Capozziello, S. D. Odintsov, "Dark energy cosmology: the equivalent description via different theoretical models and cosmography tests", Astrophys. Space Sci., 342:1 (2012), 155-228.

[7] R. M. Wald, "Black hole entropy is the Noether charge", Phys. Rev. D, 48:8 (1993), R3427-R3431, arXiv: gr-qc/9307038.

[8] N. Radicella, D. Pavón, "The generalized second law in universes with quantum corrected entropy relations", Phys. Lett. B, 691:3 (2010), 121-126.

[9] M. Jamil, A. Sheykhi, M. U. Farooq, "Thermodynamics of interacting entropy-corrected holographic dark energy in a non-flat FRW universe", Internat. J. Modern Phys. D, 19:11 (2010), 1831-1842.

[10] A. Ashtekar, J. Baez, A. Corichi, K. Krasnov, "Quantum geometry and black hole entropy", Phys. Rev. Lett., 80:5 (1998), 904-907, arXiv: gr-qc/9710007.

[11] C. Rovelli, "Black hole entropy from loop quantum gravity", Phys. Rev. Lett., 77:16 (1996), 3288-3291.

[12] A. Ghosh, P. Mitra, "Log correction to the black hole area law", Phys. Rev. D, 71:2 (2005), 027502, 3 pp.

[13] A. J. M. Medved, E. C. Vagenas, "When conceptual worlds collide: the generalized uncertainty principle and the Bekenstein-Hawking entropy", Phys. Rev. D, 70:12 (2004), 124021, $5 \mathrm{pp}$.

[14] K. A. Meissner, "Black-hole entropy in loop quantum gravity", Class. Quantum Grav., 21:22 (2004), 5245-5251.

[15] R.M. Wald, General Relativity, University of Chicago Press, Chicago, IL, 1984.

[16] A. Sheykhi, M. Jamil, "Power-law entropy corrected holographic dark energy model", Gen. Relativ. Gravit., 43:10 (2011), 2661-2672, arXiv: 1011.0134.

[17] R. Banerjee, B. R. Majhi, "Quantum tunneling and back reaction", Phys. Lett. B, 662:1 (2008), 62-65; R. Banerjee, B. R. Majhi, "Quantum tunneling beyond semiclassical approximation", JHEP, 06 (2008), 095, 20 pp.; J. Zhang, "Black hole quantum tunnelling and black hole entropy correction", Phys. Lett. B, 668:5 (2008), 353-356.

[18] Y.-F. Cai, J. Liu, H. Li, "Entropic cosmology: a unified model of inflation and late-time acceleration", Phys. Lett. B, 690:3 (2010), 213-219.

[19] H. Wei, "Entropy-corrected holographic dark energy", Commun. Theor. Phys., 52:4 (2009), $743-749$.

[20] A. Sheykhi, "Interacting holographic dark energy in Brans-Dicke theory", Phys. Lett. B, 681:3 (2009), 205-209.

[21] P. Jordan, Schwerkraft und Weltall, Friedrich Vieweg und Sohn, Braunschweig, 1955.

[22] C. Brans, R. H. Dicke, "Mach's principle and a relativistic theory of gravitation", Phys. Rev., 124:3 (1961), 925-935.

[23] C. Wetterich, "Cosmology and the fate of dilatation symmetry", Nucl. Phys. B, 302:4 (1988), 668-696; B. Ratra, P. J. E. Peebles, "Cosmological consequences of a rolling homogeneous scalar field", Phys. Rev. D, 37:12 (1988), 3406-3427.

[24] T. Chiba, T. Okabe, M. Yamaguchi, "Kinetically driven quintessence", Phys. Rev. D, 62:2 (2000), 023511, 8 pp.; C. Armendariz-Picon, V. Mukhanov, P. J. Steinhardt, "Essentials of k-essence", Phys. Rev. D, 63:10 (2001), 103510.

[25] R. R. Caldwell, "A phantom menace? Cosmological consequences of a dark energy component with super-negative equation of state", Phys. Lett. B, 545:1-2 (2002), 23-29; S. Nojiri, S. D. Odintsov, "Quantum de Sitter cosmology and phantom matter", Phys. Lett. B, 
562:3-4 (2002), 147-152; "de Sitter brane universe induced by phantom and quantum effects", 565:1 (2003), 1-9.

[26] A. Sen, "Rolling tachyon", JHEP, 04 (2002), 048, 18 pp.; T. Padmanabhan, T. R. Chodhury, "Can the clustered dark matter and the smooth dark energy arise from the same scalar field?", Phys. Rev. D, 66:8 (2002), 081301, 4 pp.

[27] E. Elizade, S. Nojiri, S. D. Odintsov, "Late-time cosmology in a (phantom) scalar-tensor theory: dark energy and the cosmic speed-up", Phys. Rev. D, 70:4 (2004), 043539, 20 pp.; S. Nojiri, S. D. Odintsov, S. Tsujikawa, "Properties of singularities in the (phantom) dark energy universe", Phys. Rev. D, 71:6 (2005), 063004, 16 pp.

[28] J. P. B. Almeida, J. G. Pereira, "Holographic dark energy and the universe expansion acceleration", Phys. Lett. B, 636:2 (2006), 75-79, arXiv: gr-qc/0602103; B. Guberina, R. Horvat, H. Nikolić, "Dynamical dark energy with a constant vacuum energy density", Phys. Lett. B, 636:2 (2006), 80-85; X. Zhang, "Dynamical vacuum energy, holographic quintom, and the reconstruction of scalar-field dark energy", Phys. Rev. D, 74:10 (2006), 103505, 7 pp.

[29] L. N. Granda, A. Oliveros, "Infrared cut-off proposal for the holographic density", Phys. Lett. B, 669:5 (2008), 275-277; "New infrared cut-off for the holographic scalar fields models of dark energy", 671:2 (2009), 199-202.

[30] X. Zhang, "Reconstructing holographic quintessence", Phys. Lett. B, 648:1 (2007), 1-7; J. Zhang, X. Zhang, H. Liu, "Agegraphic dark energy as a quintessence", Eur. Phys. J. C, 54:2 (2008), 303-309; X. Zhang, "Holographic Ricci dark energy: current observational constraints, quintom feature, and the reconstruction of scalar-field dark energy", Phys. Rev. D, 79:10 (2009), 103509, 12 pp.

[31] K. Karami, M. S. Khaledian, F. Felegary, Z. Azarmi, "Interacting new agegraphic tachyon, K-essence and dilaton scalar field models of dark energy in non-flat universe", Phys. Lett. B, 686:4-5 (2010), 216-220.

[32] M. R. Setare, "The holographic dark energy in non-flat Brans-Dicke cosmology", Phys. Lett. B, 644:2-3 (2007), 99-103; "Interacting holographic dark energy model in non-flat universe", 642:1-2 (2006), 1-4; "Holographic Chaplygin gas model", 648:5-6 (2007), 329-332; "Holographic tachyon model of dark energy", 653:2-4 (2007), 116-121; "Interacting holographic generalized Chaplygin gas model", 654:1-2 (2007), 1-6; "Interacting holographic phantom", Eur. Phys. J. C, 50:4 (2007), 991-998; M. R. Setare, J. Zhang, $\mathrm{X}$. Zhang, "Statefinder diagnosis in a non-flat universe and the holographic model of dark energy", JCAP, 03 (2007), 007, 16 pp.; M. R. Setare, E. C. Vagenas, "The cosmological dynamics of interacting holographic dark energy model", Internat. J. Modern Phys. D, 18:1 (2009), 147-157.

[33] P. J.E. Peebles, B. Ratra, "The cosmological constant and dark energy", Rev. Modern Phys., 75:2 (2003), 559-606.

[34] M. Arik, M. Çalik, "Can Brans-Dicke scalar field account for dark energy and dark matter?", Modern Phys. Lett. A, 21:15 (2006), 1241-1248.

[35] M. Jamil, K. Karami, A. Sheykhi, E. Kazemi, Z. Azarmi, "Holographic dark energy in Brans-Dicke cosmology with Granda-Oliveros cut-off", Internat. J. Theor. Phys., 51:2 (2012), 604-611; N. Banerjee, D. Pavón, "Holographic dark energy in Brans-Dicke theory", Phys. Lett. B, 647:5-6 (2007), 477-481.

[36] H. Kim, "Brans-Dicke theory as a unified model for dark matter-dark energy", Mon. Not. Roy. Astron. Soc., 364:3 (2005), 813-822.

[37] Y.-Z. Ma, Y. Gong, X. Chen, "Features of holographic dark energy under combined cosmological constraints", Eur. Phys. J. C, 60:2 (2009), 303-315; C. Feng, B. Wang, Y. Gong, R.-K. Su, "Testing the viability of the interacting holographic dark energy model by using combined observational constraints", JCAP, 09 (2007), 005, 15 pp.; Q. Wu, Y. Gong, A. Wang, J. S. Alcaniz, "Current constraints on interacting holographic dark energy", Phys. Lett. B, 659:1-2 (2008), 34-39. 
[38] J. Lu, W. Wang, L. Xu, Y. Wu, "Does accelerating universe indicate Brans-Dicke theory?", Eur. Phys. J. Plus, 126:10 (2011), 92, 13 pp.

[39] A. Sheykhi, K. Karami, M. Jamil, E. Kazemi, M. Haddad, "Holographic dark energy in Brans-Dicke theory with logarithmic correction", Gen. Relativ. Gravit., 44:3 (2012), 623-638.

[40] Q.-G. Huang, M. Li, "The holographic dark energy in a non-flat universe", JCAP, 08 (2004), 013, 10 pp.

[41] A. Pasqua, I. Khomenko, "Interacting Ricci logarithmic entropy-corrected holographic dark energy in Brans-Dicke cosmology", Internat. J. Theoret. Phys., 52:11 (2013), 3981-3993, arXiv: 1212.2157. 